\title{
Simple model of recovery dynamics after mass extinction
}

\author{
Ricard V. Solé ${ }^{1,2}$, Joan Saldaña ${ }^{3}$, Jose M. Montoya ${ }^{1,4}$ and Douglas H. Erwin ${ }^{2,51}$ \\ 11 ICREA-Complex Systems Lab, Universitat Pompeu Fabra, Dr. Aiguader 80, 08003 Barcelona, Spain \\ ${ }^{2}$ Santa Fe Institute, 1399 Hyde Park Road, New Mexico 87501, USA \\ ${ }^{3}$ Departament d'Informàtica i Matemàtica Aplicada, Universitat de Girona, 17071 Girona, Spain \\ 4 Department of Marine Biology and Oceanography, CMIMA-CSIC, \\ Passeig Martim de la Barceloneta 37-49. 08003 Barcelona \\ ${ }^{5}$ Department of Paleobiology, MRC-121 National Museum of Natural History, Washington, D.C. 20560 USA
}

Biotic recoveries following mass extinctions are characterized by a complex set of dynamics, including the rebuilding of whole ecologies from low-diversity assemblages of survivors and opportunistic species. Three broad classes of diversity dynamics during recovery have been suggested: an immediate linear response, a logistic recovery, and a simple positive feedback pattern of species interaction. Here we present a simple model of recovery which generates these three scenarios via differences in the extent of species interactions, thus capturing the dynamical logic of the recovery pattern. The model results indicate that the lag time to biotic recovery increases significantly as biotic interactions become more important in the recovery process.

Keywords:

\section{INTRODUCTION}

Until recently paleontologists have focused on understanding the dynamics of the extinction process and assumed that with the amelioration of the causes of the extinction recovery would be straightforward: diversification of new species and migration of surviving species would ensure rapid repopulation of niches vacated by the extinction. Little attention was paid to the actual dynamics of recovery. Logistic models derived from ecology were commonly seen as the appropriate theoretical framework (e.g. Sepkoski 1978, 1979,1984), coupled with the dynamics of diversification into empty niches (Valentine 1980; Valentine and Walker 1986), where the rate of diversification was simply assumed to be diversity dependent. However the lag of some 4 million years between the end of the end-Permian mass extinction and the onset of recovery of most marine and terrestrial ecosystems (Erwin 1998a, 1998b; 2007; Sahney and Benton 2008) and the presence of many 'Lazarus' taxa which disappear from the fossil record before the extinction but then reappear late in the recovery, suggests something of the complex ecological and evolutionary dynamics of postextinction intervals.

As paleontologists have begun to explore these complex dynamics over the past decade most have followed a largely empirical approach, documenting local and regional recovery patterns, exploring the structure of recovery communities and investigating the correlation to changes in stable isotopes (e.g. d'Hondt et al 1998; Payne et al., 2004). As valuable as such data is, increased understanding of the dynamics of post-extinction diversification also requires an appropriate theoretical frame- work, both for understanding and interpreting existing data and for guiding new research. Recent efforts in this direction include the explicit modeling of recovery dynamics using trophic network models (Solé et al., 2002a; Roopnarine 2006; Roopnarine et al., 2007; see also Solé and Bascompte, 2006; Yedid et al., 2009). These models consider some sort of trophic interaction pattern between species belonging to different levels. The number of such levels, their species composition as well as the number and strength of links evolve with speciation and extinction.

Here we consider three different recovery scenarios: First, immediate biotic recovery following a mass extinction, modeled as a linear response. This appears similar to at least two events in the fossil record, the endOrdovician mass extinction about 439 million years ago (Ma) and possibly the end-Triassic mass extinction 199 Ma. This may also apply to some smaller biotic crises, and roughly corresponds to the standard description of so called neutral communities (e.g. Hubbell 2001; Alonso et al., 2006), where the intensity of species interactions do not affect the recovery process. Second, a logistic expansion, which is the traditional expectation of paleontologists (e.g. Sepkoski, 1984). It is not clear that there are good empirical examples of this pattern.

Third, we also present a simple model of diversification driven by biotic interactions between pairs of species. This model is a first effort at examining the relevance of positive feedback processes in evolutionary diversifications and provides well-defined predictions of the time lags to recovery that should be expected. In each of the models we consider the equilibrium case where diversity returns to the pre-extinction level. In other words, the 

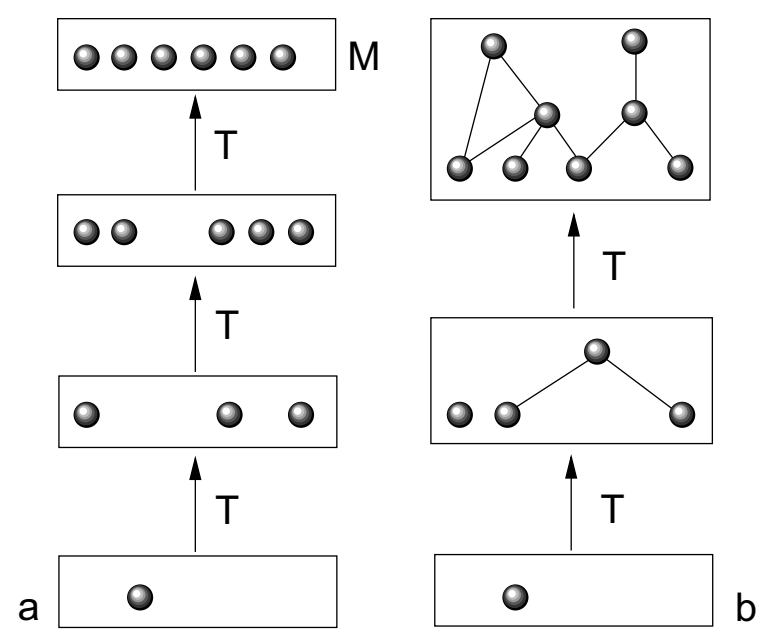

FIG. 1: Recovery patterns. Recoveries might essentially consist of refilling available space (until some carrying capacity $M$ is reached) as indicated in (a). But they also might require ecosystem rebuilding (b) relying in the reconstruction of lost interactions, functionality and other attributes.

maximal diversity in the system is pre-determined. In reality of course, the most interesting cases are those where post-extinction diversity increases.

Identifying the pattern of recovery might help to establish the relevance of biotic interactions in rebuilding functional ecosystems at evolutionary scales. In a related ecological context, recent attempts to explain current patterns of biodiversity at local and regional scales (e.g. Hubbell 2001) assumed ecosystems as neutral entities, where interactions among species are not important. This perspective has recently being challenged (Wooton 2005; seealso Pueyo 2006 and Pueyo et al), showing that neutral models predicts poorly field experimental results, indicating and essential role of species interactions. In this context, more general models can be defined by expanding the neutral approach and considering different levels of heterogeneous interactions (Solé et al., 2002b).

The results obtained from our model suggest that the greater the interactions among pairs of existing species in the generation of new species, the longer the delay in recovery. The long delay together with a rapid rise of diversity could result from two different processes: (a) increasing numbers of ecological interactions might provide the context for new opportunities to speciate, but building new links is a slow, costly process, strongly dependent on external and internal constraints. Alternatively, (b) as the number of species and thus interaction grows, the potential combinations eventually may allow a rapid increase in species. Our approach is the simplest one able to capture these two ingredients.

\section{MATHEMATICAL MODEL}

The simplest model able to cope with biotic recovery would be a logistic one (Sepkoski, 1984). Here, if $S$ indicates the number of species and $\mu$ is speciation rate, we have:

$$
\frac{d S}{d t}=\mu S\left(1-\frac{S}{M}\right)
$$

where $M$ is the maximum number of allowed species. In classical population dynamics (Case, 1999) this maximum is the so called carrying capacity (usually indicated as $K$ ) and gives the maximal number of individuals, whereas $\mu$ would be the per capita growth rate. Here speciation is defined as the difference $\mu=o-e$ between origination $(o)$ and extinction (e) rates. Specifically, as described by Sepkoski (1984) these are per-taxon rates and are expected to be stochastic parameters. In a deterministic context (to be followed here) these parameters are considered average values roughly constant over (geologic) time.

This model however is unable to explain the spectra of recovery patterns observed in the fossil record even if we widely vary the model parameters. The reason is that the underlying assumptions involve a system in which ecological interactions are solely based on the available resources, with no explicit interaction among species. The situation is schematically drawn in figure 1(a), where species are indicated as gray balls and they monotonously fill the available space.

One way of generalizing the previous approach is to consider a dependence of speciation dynamics on species interactions. The underlying assumption, schematically drawn in figure 1 (b) is that post-extinction biotic recovery (and indeed ecosystem rebuilding in general) is not described by means of a neutral process. Instead, it deals with the full spectrum of biotic interactions displayed by species belonging to different trophic levels and having different ecological interactions, from mutualism to parasitism. In such a context, the opportunities available to a given species to speciate are dependent upon the biotic structure of the current community. Actually, some general patterns exhibited by complex ecosystems over evolutionary time scales, such as community rebuilding after a major extinction event are closely related to some phenomena occuring at short, ecological time scales, such as ecological succession (Solé et al., 2002; Solé and Bascompte 2006).

We can make such approach explicit by considering three basic scenarios of diversity increase. The logic of these scenarios is sketched in figure $2 \mathrm{a}-\mathrm{c}$. We will reduce our description to a single macroscopic variable $S$ (number of species) and a single free parameter $\mu$ representing speciation rate. The three diagrams indicate how these two ingredients influence each other. Here we do not indicate explicitely the negative feedback associated with limited resources. As will be shown below, these three plots can be directly translated into explicit equa- 
a

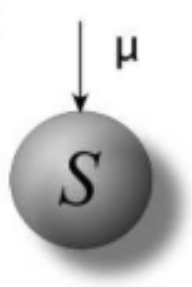

$\beta=0$ b

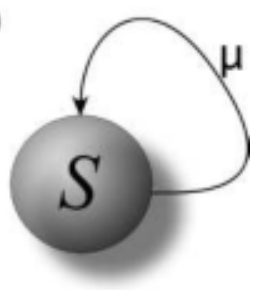

$\beta=1$

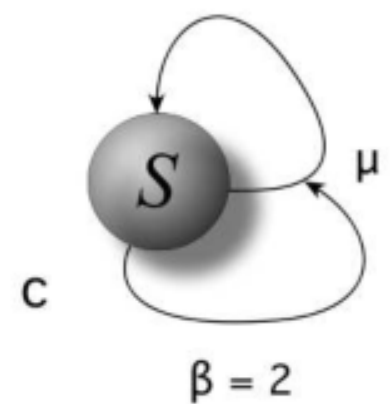

FIG. 2: Three possible mechanistic scenarios for recovery. Here $S$ indicates the number of species and $\mu$ speciation rates. In (a) a constant, diversity-independent rate is at work. In (b) speciation rate is species-dependent: the more species present the more species are generated. The third case (c) involves a positive feedback where the presence of species also enhances speciation rates.

tions for diversity dynamics. In order to further define our three models, let us consider a more microscopic description. If " 0 " denotes an empty niche (allowed to exist provided that resources are available) and $S_{i}$ corresponds to a given species $i$, it is possible to describe the three previous possibilities in terms of three simple transitions among states. These are:

$$
\begin{gathered}
0 \stackrel{\mu}{\longrightarrow} S_{i} \\
0+S_{i} \stackrel{\mu}{\longrightarrow} S_{i}+S_{i}^{\prime} \\
0+S_{i}+S_{j} \stackrel{\mu}{\longrightarrow} S_{i}+S_{i}^{\prime}+S_{j}
\end{gathered}
$$

where each of these transitions occurs at a rate $\mu$. The first case would correspond to figure $2 \mathrm{a}$, a situation in which empty niches are simply refilled by invading species and thus $\mu$ effectively represents immigration rate. The second (figure 2b) implies the presence of empty niche and an available species (here indicated as $S_{i}$ ) in order to speciate and obtain a new species (here indicated as $\left.S_{i}^{\prime}\right)$. Finally, if species $S_{i}$ requires species $S_{j}$ to generate a new species (since $S_{i}$ is, say, a parasite of species $S_{j}$ ) then the two of them are necessary to effectively obtain an speciation event (figure 2c).
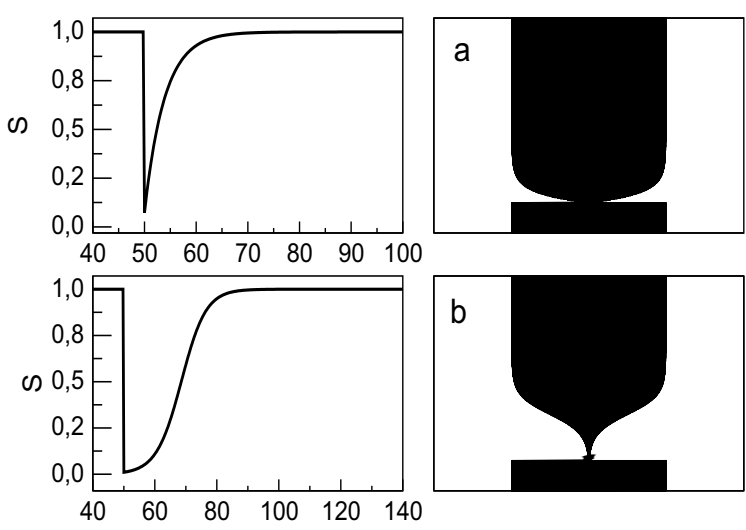

b

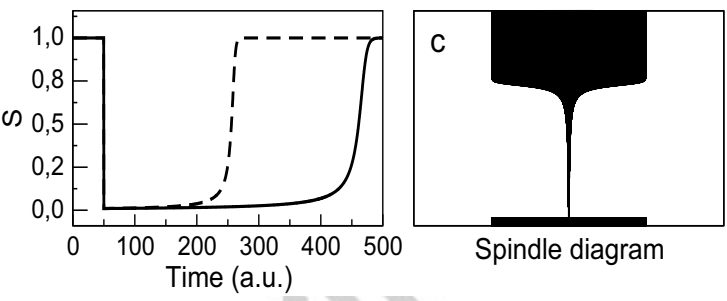

FIG. 3: Recovery patterns under each of the three scenarios of the model, involving different types of ecological interactions: (a) $\beta=0$, (b) $\beta=1$ and (c) $\beta=2$. Both the diversity over time (left) and the spindle diagrams (right) are shown. Here we have used $\mu=1$ (continuous lines) and $\mu=0.5$ (dashed lines, (c)). The spindle diagram for the third case corresponds to the later value.

In order to take into account the three situations, the previous equation (1) can be generalized as:

$$
\frac{d S}{d t}=\mu \Phi_{\beta}(S)\left(1-\frac{S}{M}\right)
$$

where the function $\Phi_{\beta}(S)$ includes the possibility of considering the key role of existing species to rebuild the ecosystem through speciation.

One of the simplest choices is a power functional form, i. e.

$$
\Phi_{\beta}(S)=S^{\beta}
$$

with $\beta \geq 0$. This choice is consistent with the previous representation of the three scenarios in terms of reactions. By considering these three case studies, the model naturally produces the three main types of recovery pattern. These correspond to:

\section{A. Linear model, $\beta=0$}

In this case we have a system in which the increase in species numbers would follow a linear equation:

$$
\frac{d S}{d t}=\mu\left(1-\frac{S}{M}\right)
$$


where available resources (such available space and nutrients) allow species diversity to linearly increase through time until the maximum is reached. Using hereafter (without loss of generality) $M=1$, it is not difficult to solve this equation, which gives an asymptotic increase:

$$
S(t)=1-\left(1-S_{0}\right) e^{-\mu t}
$$

This corresponds to a situation in which species coming from an external (biogeographic) pool invade the area of interest. Examples would include re-forestation after a devastating forest fire or re-population following a regionally extensive volcanic eruption. The resulting pattern (figure 3a) shows a monotonous increase on diversity takes place promtly reaching pre-extinction levels. In this figure (right column) we also show the corresponding spindle diagram, which provides a complementary picture of the diversification process, showing how relative diversity expands or shrinks through (geological) time.

This model belongs to the class of models used in island biogeography, where islands free of species are colonized by the mainland pool. Such colonization process is typically assumed to be a linear function of the species abundances. Similarly, the extinction rate is also considered a constant process.

\section{B. Density-dependent, $\beta=1$}

This corresponds to the logistic case (Sepkoski, 1984). Species essentially interact because of their limiting resources (i.e. they compete), and no further biotic interactions are involved. The solution to (5) with $M=1$ now reads:

$$
S(t)=\left[1+\left(\frac{1-S_{0}}{S_{0}}\right) e^{-\mu t}\right]^{-1}
$$

again asymptotically reaching the carrying capacity. The two basic trends present in logistic growth (initial exponential increase followed by saturation) are clearly observed in figure $3(\mathrm{~b})$.

\section{Hyperbolic case, $\beta=2$}

This scenario considers the interaction among pairs of existing species. It assumes that speciation requires pairs of species to be interacting, not only through competition. This requirement strongly constrains the speed of speciation events, since interactions are necessary to evolve (co-evolve). A given parasite, for example, needs a host to reproduce. The presence of such a pair is also needed for the parasite to evolve new traits and eventually lead to a new species. A similar argument can be made with predator-prey or mutualistic interactions. The dynamics are now governed by:

$$
\frac{d S}{d t}=\mu S^{2}(1-S)
$$

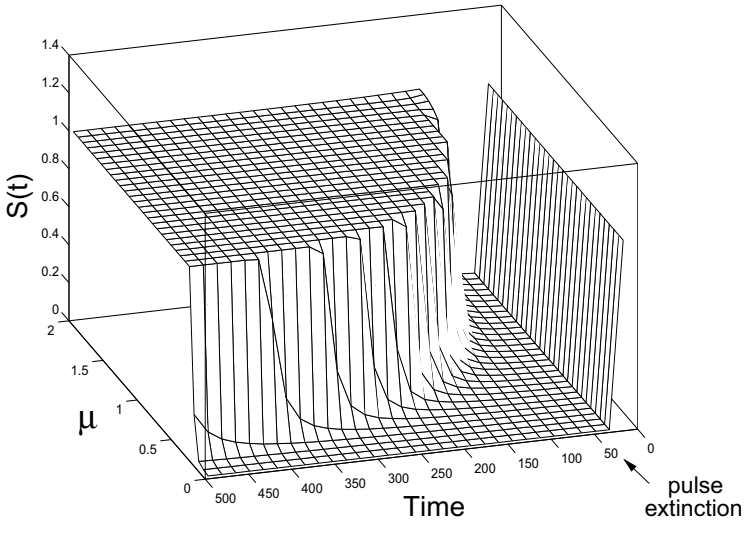

FIG. 4: Recovery pattern for the hyperbolic $(\beta=2)$ model. Here a pulse extinction occurs at time zero. The initial diversity after the pulse extinction is $S_{0}=0.01$ and speciation rates are varied in a given range $(2 \geq \mu \geq 0)$. There is always a delay, even when large rates are used, although it becomes shortened in a nonlinear way. Here equation (10) has been numerically integrated using Euler's method

superficially resembling the logistic equation. However, the feedback implicit on the dependence of speciation on interactions among species leads to a very different dynamical behavior, characterized by a long lag after a rapid, strong perturbation is performed on the system (Erwin, 1996) and is illustrated in figure 3(c), where two different speciation rates have been used. The long delay is clearly apparent in both cases. This situation corresponds to hyperbolic replicator dynamics, similar to the one displayed by models of hypercyclic organization (Szathmáry and Maynard Smith, 1997). In figure 4 we show the effects of continuously varying speciation rates on the time evolution of species numbers after the pulse extinction event.

This hyperbolic growth model has been used to describe the dynamics of marine biodiversity through the Phanerozoic (Markov and Korotayev, 2007) and seems to better fit available data than the exponential one (see Benton, 1995). Such type of model has also been applied to the dynamics of world population and economics (Johansen and Sornette, 2001 and references cited).

In general, for any $\beta \geq 0$ and if $S_{0}<1$, the solution $S(t) \in(0,1)$ for any $t>0$. So, when comparing growth rates of the different solutions with the same initial condition $S_{0}$, it follows

$$
\left.\frac{d S}{d t}\right|_{\beta<1}>\left.\frac{d S}{d t}\right|_{\beta=1}>\left.\frac{d S}{d t}\right|_{\beta>1},
$$

where the derivatives are evaluated at the same moment. In particular, it follows that the growth rate of the number of species (i.e. diversification rate) according to the model with $\beta>1$ is very much lower than the the logistic case $(\beta=1)$ as long as $S(t) \ll 1$, i.e., when the 


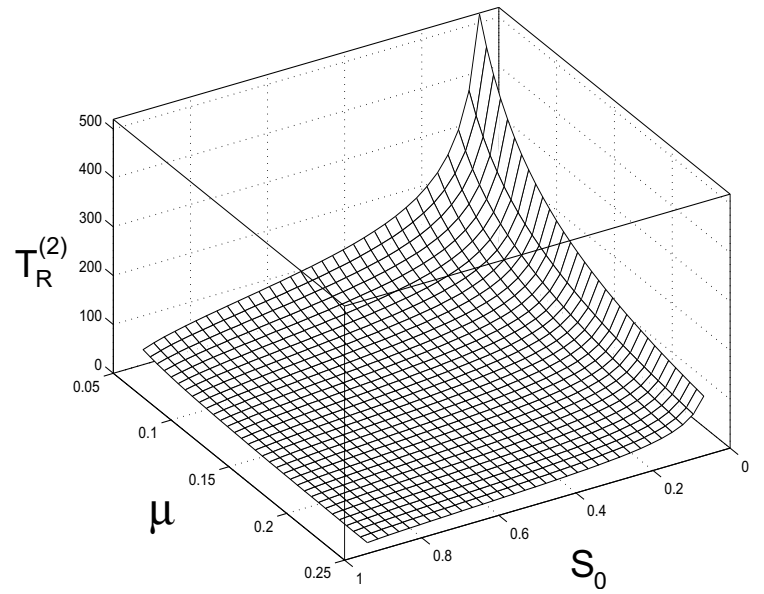

FIG. 5: Characteristic delay to recovery based on the approximate solution given by $T_{R}^{(2)}$ (equation 19). The delay time quickly decays with increasing speciation or decreasing pulse size perturbation.

community is starting to define their biotic interactions. Therefore, $\beta$ reflects the dependence of diversification rates on species interactions. In other words, how fast diversity will increase from a given initial condition (such as immediately after a major extinction) if $\beta$-dependent. Larger $\beta$ values imply slower speeds of diversity growth (as indicated by the smaller derivatives) thus suggesting that perhaps the tempo and mode of recovery dynamics is strongly influenced by the ecological constraints associated to speciation events. If true, this should be observed in terms of different types and durations of recovery times, as shown in the next section.

\section{DELAY TIMES TO RECOVERY}

One of the fundamental questions relating recovery patterns involves the time lags to be expected until complete recovery is reached. As we noted before, the most interesting case occurs when total diversity is unconstrained in a non-equilibrium system, but here we continue to examine the restricted case where an equilibrium diversity is defined. Moreover, speciation rates must strongly influence the speed of recovery. This is illustrated in figure 4 for the $\beta=2$ case using different speciation rates. As can be appreciated, a wide range of delay times is observable. In order to compare them, let us assume that the initial condition $S_{0}$ is the same for all models and satisfies $S_{0} \ll 1$ (that corresponds to the condition after a mass extinction), and let us fix a value $S^{*}$ close to (but less than) the carrying capacity. Our goal here is to obtain well-defined estimates of recovery delay times $T_{R}^{(\beta)}$ for different $\beta$ values.
For $\beta=0$, we have, from equation (7) and $M=1$,

$$
T_{R}^{(0)}=\frac{1}{\mu} \int_{S_{0}}^{S^{*}} \frac{d S}{1-S}
$$

which gives after integration to a delay time:

$$
T_{R}^{(0)}=\frac{1}{\mu} \log \left(\frac{1-S_{0}}{1-S^{*}}\right)
$$

Similarly, the recovery time for the logistic model $(\beta=$ 1) can be easily obtained. By integrating equation (1) with $M=1$, we obtain

$$
\mu t=\log \left(\frac{1-S_{0}}{1-S(t)}\right)+\log \left(\frac{S(t)}{S_{0}}\right)
$$

and so the recovery time for this case will be

$$
T_{R}^{(1)}=\frac{1}{\mu}\left[\log \left(\frac{1-S_{0}}{1-S^{*}}\right)+\log \left(\frac{S^{*}}{S_{0}}\right)\right]
$$

A similar calculation can be performed for $\beta=2$, which gives a recovery time

$$
T_{R}^{(2)}=\frac{1}{\mu}\left[\log \left(\frac{1-S_{0}}{1-S^{*}}\right)+\log \left(\frac{S^{*}}{S_{0}}\right)+\frac{1}{S_{0}}-\frac{1}{S^{*}}\right]
$$

The recovery time for the general model with a given integer $\beta \geq 2$ is given by:

$$
T_{R}^{(\beta)}=T_{R}^{(1)}+\frac{1}{\mu} \Gamma\left(S_{0}, S^{*}, \beta\right)
$$

where $\Gamma\left(S_{0}, S^{*}, \beta\right)$ is given by the sum:

$$
\Gamma\left(S_{0}, S^{*}, \beta\right)=\sum_{m=1}^{\beta-1} \frac{\left(S^{*}\right)^{m}-S_{0}^{m}}{m S_{0}^{m}\left(S^{*}\right)^{m}}
$$

These previous expressions can be simplified under the previous assumptions on $S_{0}$ and $S^{*}$, namely,

$$
1-S^{*} \sim O\left(S_{0}\right)
$$

with $S_{0} \ll 1$, that is, assuming a large extinction event and an almost full recovery of diversity. In this case, we have the following approximate estimations for delay times $T_{R}^{(i)}$

$$
\begin{gathered}
T_{R}^{(0)} \approx-\frac{\log S_{0}}{\mu}, \\
T_{R}^{(1)} \approx-\frac{2 \log S_{0}}{\mu}, \\
T_{R}^{(2)} \approx \frac{1}{\mu S_{0}},
\end{gathered}
$$


and, for the general model with $\beta \geq 2$,

$$
T_{R}^{(\beta)} \approx \frac{1}{\mu(\beta-1) S_{0}^{\beta-1}} .
$$

It is easy to compare the previous estimates and find that the following inequalities hold:

$$
T_{R}^{(2)} \gg T_{R}^{(1)} \sim 2 T_{R}^{(0)}
$$

in other words, recovery times rapidly increase as biotic interactions become more important within a community. Although lag times are comparable under scenarios not considering species interactions, the situation sharply changes at $\beta=2$. This result confirms the expectation of a qualitatively different pattern when recovery relies upon ecosystem rebuilding, comprising both the presence of species and their interactions, The simplicity and generality of our analytic expressions allow to test our predictions with real data.

The relationship between delay times changes with both the size of the extinction event and the rate of speciation in the hyperbolic case (figure 5). The theory predicts a rapid decay in $T_{R}^{(2)}$ inversely proportional to $\mu$ as well as a rapid increase with larger extinction events.

\section{DISCUSSION}

Mass extinctions have received considerable attention from paleontologists, evolutionary biologists and others over the past several decades (see Jablonski, 2005 and references therein). The subsequent process of biotic recovery has received much less attention, in part we think, because of the assumption that once the cause of the extinction was ameliorated the survivors would quickly respond. The growing number of studies of biotic recoveries (Erwin 1996, 1998a, 2001; Benton and Twitchett, 2003; Lockwood, 2004; Wagner et al., 2006; Payne et al, 2006; Aberhan et al., 2007) suggest more complex dynamics are occurring. Although paleontologists once assumed (often tacitly) that survivors simply diversified into the empty ecospace produced by the mass extinction (see Erwin 1993 for discussion) the biogeographic variability in recovery rates and processes (e.g. Jablonski 1998), as well as the recognition that some clades may survive the extinction only to succumb during the subsequent radiation, indicates that more complex processes may be operating. In addition, from theoretical grounds we would expect clades at different trophic levels (e.g. producers, herbivores, carnivores) and functional groups (e.g. grazers, detritivores) to respond at different rates.

In this paper we have taken a theoretical approach to the problem of recovery based on the simplest and most general approximation that can be taken. Without considering the particularities of the underlying ecological network, we have explored the consequences of including (or not) pairwise species interactions as part of the constraints involved in generating new species. Each of three
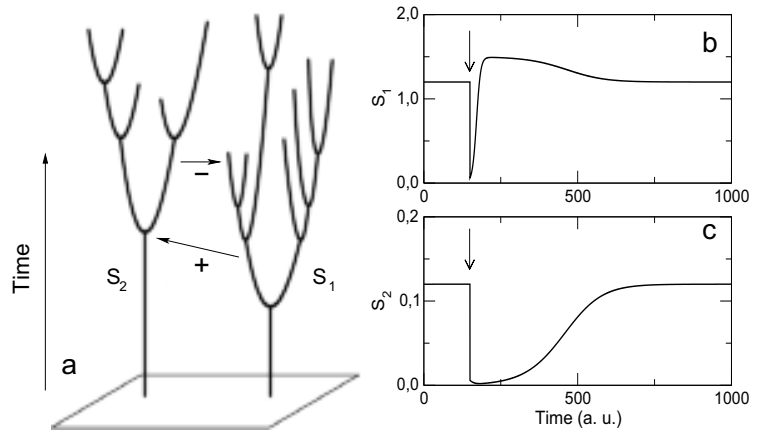

FIG. 6: Patterns of recovery in a complex ecosystems involving two tropic levels (producers and consumers). In (a) a schematic diagram shows that the philogenetic branching associated to consumers (left tree) and producers (right tree) are linked through the presence of ecological interactions of different signs. Speciation within the producers trigger speciation in consumers (positive interaction) whereas increasing numbers of consumer species might eventually reduce the diversity of producers (negative arrow). A simple mathematical model (equations 22-23) illustrates the different nature of the recovery patterns for the two levels (see text). Parameters chosen here: $M=1.5, \mu=0.1, \delta=0.25, \epsilon=0.05, e=0.06$. Recovery patterns for the two trophic levels are different (bc).

recovery scenarios predicted by the model presented here may characterize different recovery periods. But they may also characterize and distinguish, within a particular period, the recovery dynamics of different taxa, trophic levels, or biogeographical areas. The rapid linear recovery of primary producers, for example, contrasts with observed delays in higher trophic levels (e.g. Hart 1996, D'Hondt et al. 1998). Within taxa, or feeding groups, omnivore and detritivore species typically recover faster than strict hervibores and carnivores (Smith and Jeffrey 1998; Fara 2000). Finally, biogeographic differences in recovery patterns (e.g. Jablonski 1986, 1998) may also reflect differences in the relevance of biotic interactions for diversification among different areas. Therefore, identifying the pace of recovery informs on the processes responsible of diversification.

There is actually a simple connection between our model, which only includes a homogeneous set of species, and models considering multiple trophic levels. As an example, let us consider a two-trophic system composed by two species assemblages: producers and primary consumers. We thus consider two simultaneously evolving groups (figure 6a) which interact through coevolving ecological links. Indicating as $S_{1}$ and $S_{2}$ the number of species at each level, a macroscopic view of the ecologically-driven evolutionary dynamics can be obtained from the following set of equations:

$$
\frac{d S_{1}}{d t}=\mu S_{1}\left(M-S_{1}\right)-\delta S_{1} S_{2}
$$




$$
\frac{d S_{2}}{d t}=\epsilon S_{1} S_{2}-e S_{2}
$$

We can see that the previous equations, describing changes in the number of species through time, are not formally different from a standard Lotka-Volterra model of consumer-producer dynamics. However, the interpretation of the parameters must be based on a different criterion: $\mu$ is again speciation rate for producers, whereas $\delta$ and $\epsilon$ need to be interpreted as the consumer-driven species extinction rate and the consumer (externallydriven) extinction rate, respectively. An example of the dynamics displayed by this model is shown in figure 6 . After a major extinction (reducing both assemblies to $5 \%$ of their pre-extinction equilibrium diversity) producers recover first, followed by consumers after a long delay (fig 6 b-c). The first group follows a rapid recovery characteristic of the simple model discussed here for $\beta \cong 1$. Consistently with our prediction, consumers need a much larger time, because the speciation rate $\epsilon S_{1} S_{2}$ that appears in the right-hand side of equation (23) is the product of two species numbers, thus consistent with the $\beta=2$ model discussed before. This observation helps interpret the meaning of our former model in light of the position of the group (e.g. trophic level) whose recovery pattern is analyzed within a given ecological network (e.g. food web). In this sense, long delays should be expected as we move up through the food chain (Solé et al., 2002).

There are two ways to achieve greater understanding of recovery dynamics. Most paleontologists have followed a largely empirical approach of developing detailed analyses of the recovery patterns within local or regional areas, sometimes integrating the fossil data with geological or geochemical information. Here the hope is that through the accumulation of a wealth of data some empirical generalities might emerge. The alternative approach is to develop, test and refine models of the processes that may be involved in recovery, to serve as a guide to empirical exploration.

The number of available models of recovery is very limited, however. As mentioned, Sepkoski (1984) invoked a simple pattern of logistic growth following the end of a biotic perturbation, although with different intrinsic rates of increase between his Evolutionary Faunas. The teserae models of Valentine (1980; see also Valentine and Walker 1986, 1987) have had a more pervasive effect. Instantiations of earlier less-rigorously formulated ideas on filling empty ecospace, they explicitly considered how clades might diversify into open ecospace. These models invoked empty niches (not simply unutilized resources) as a critical component and did not examine how species interactions might facilitate diversification. These models, strongly influenced by the ecology of that time (MacArthur and Levins 1967, Rosenzweig 1975) considered competition between species as the only interaction regulating diversity recoveries, which inextricably leads to logistic recovery patterns. However, other types of interactions, i.e. facilitation, mutualism or predation, played a crucial role in the rebuilding process and add complexity to the diversification process. Indeed, current ecological models of filling niche space assume species' niches that are partially determined by their interactions with other species within the community, both as resources and as consumers, and speciation occurs within this ecological context where network structure plays a key role (Williams \& Martinez 2000, Drossel et al. 2004; Pascual and Dunne 2004; Montoya et al., 2006; Dunne et al. 2008).

We began developing a new generation of recovery models several years ago. Initially (Sole et al 2002) we evaluated a simple model with three trophic levels corresponding to primary producers, herbivores and carnivores and found that recovery would initiate at the lowest level of the food chain, and delays in recovery times increased as we move up in the food web. These results immediately suggested that empirical studies should investigate the relative timing of post-extinction recoveries at different trophic levels, using carbon isotope fluctuations as a proxy for primary productivity (e.g. d'Hondt et al 1998).

More useful, however, would be a suite of process-based models, testable with evidence from the fossil record, which would capture the range of possible post-extinction recovery dynamics (Roopnarine et al., 2007). This would allow paleontologists to plan field work with the intent of testing these alternative models and allow iterative refinement of such models. With this paper we have begun development of such a theoretical toolkit, with the first process-based model to capture species interactions as an essential component. The results make several specific predictions that can be further tested with data from different recovery events. In particular, if species interactions are a significant component of post-extinction diversification then the length of the delay before the onset of rapid recovery should scale with the speciation rate.

\section{Acknowledgments}

The authors thank the members of the complex systems lab for useful discussions. This work has been supported by a grant FIS 2004-05442 (RVS), the McDonnell Foundation (RVS), the Santa Fe Institute (RVS and DHE) and the Thaw Cheritable Trust (DHE).

\section{REFERENCES}

1. Aberhan, M., S. Weidemeyer, W. Kiessling, R. A. Scasso, and F. A. Medina. 2007. Faunal evidence for reduced productivity and uncoordinated recovery in Southern Hemisphere Cretaceous-Paleogene boundary sections. Geology 35:227-230.

2. Alonso, D., Etienne, R. S. and McKane, A. J. 2006. The merits of neutral theory. Trends Ecol. Evol. $21,351-356$. 
3. Benton, M. J. 1995.

4. Benton, M. J. and Twitchett, R. J. 2003. How to kill (almost) all life: the end-Permian extinction event. Trends Ecol. Evol. 18: 358-365.

5. Case, T.J. 2000. An illustrated guide to theoretical ecology. Oxford U. Press, Oxford.

6. D'Hondt, S., Donaghay, P., Zachos, J.C., Luttenberg, D. and Lindinger, M. 1998. Organic carbon fluxes and ecological recovery from the CretaceousTertiary mass extinction. Science 282: 276-279.

7. Drossel, B., McKane, A.J. and Quince, C. 2004. The impact of non-linear functional responses on the long-term evolution of food web structure. J. Theoretical Biology 229: 539-548.

8. Erwin, D. H. 1993. The Great Paleozoic Crisis: Life and Death in the Permian. Columbia U. Press.

9. Erwin, D. H. 1996. Understanding biotic recoveries: extinction, survival and preservation during the end-Permian mass extinction. pp. 398-418. In D. Jablonski, D. H. Erwin, and J. H. Lipps, eds. Evolutionary Paleobiology. University of Chicago Press, Chicago.

10. Erwin, D. H. 1998a. The end and the beginning: recoveries from mass extinctions. Trends Ecol. Evol. 13: 344-349.

11. Erwin, D. H. 1998b. After the end: recovery from mass extinction. Science 279: 1324-1325.

12. Erwin, D. H. 2001. Lessons from the past: biotic recoveries from mass extinction. Proc. Natl. Acad. Sci. USA 98(10): 5399-403.

13. Erwin, D. H. 2007. Increasing returns, ecological feedback and the Early Triassic recovery. Paleoworld 16, 9-15.

14. Erwin, D. H. 2008. Extinction and the loss of evolutionary history. Proc. Natl. Acad. Sci. USA $105,1152-11527$.

15. Fara, E. (2000) Diversity of Callovian-Ypresian (middle Jurassic-Eocene) tetrapod families and selectivity of extinctions at the $\mathrm{K} / \mathrm{T}$ boundary. Geobios 33, 387-396.

16. Hart, M.B. 1996. Recovery of the food chain after the Late Cenomanian extinction event. In Biotic recovery from mass extinction events (M.B. Hart, ed.), pp. 265-277. Geological Society Special Publication.

17. Hubbel, S.P. 2001. The Unified Theory of Biodiversity and Biogeography. Princeton University Press, Princeton.
18. Jablonski, D. 1986. Background and mass extinction: The alternation of macroevolutionary regimes. Science 231: 129-133.

19. Jablonski, D. 1998. Geographic variation in the molluscan recovery from the End-Cretaceous extinction. Science 279: 1327-1330.

20. Jablonski, D. 2005. Mass extinctions and macroevolution. Paleobiology 31(2): 192-210.

21. Lockwood, R. 2004. The K/T event and infaunality: morphological and ecological patterns of extinction and recovery in veneroid bivalves. Paleobiology 30:507-521.

22. MacArthur, R.H. Levins, R. 1967. The limiting similarity, convergence, and divergence of coexisting species. American Naturalist 101: 377-385.

23. Miller, A. I. 1998. Biotic transitions in global marine diversity. Science 281: 1157-1160.

24. Montoya, J. M., Pimm, S. L. and Sol, R. Ecological networks and their fragility. Nature 442, 259-264.

25. Payne, J. L., Lehrmann, D. J., Wei, J., Orchard, M. J., Schrag, D. P., and Knoll, A. H. (2004) Large perturbations of the carbon cycle during recovery from the end-Permian extinction. Science 305: 506509.

26. Payne, J. L., D. J. Lehrmann, S. Christensen, J. Y. Wei, and A. H. Knoll. 2006. Environmental and biological controls on the initiation and growth of a Middle Triassic (Anisian) reef complex on the Great Bank of Guizhou, Guizhou Province, China. Palaios 21:325-343.

27. Pueyo, S. 2006.

28. Pueyo, S., He, F. and Zillio, T. 2007. The maximum entropy formalism and the idiosyncratic theory of biodiversity. Eco. Lett. 10, 1017-1028.

29. Roopnarine, P. D. 2006. Extinction cascades and catastrophe in ancient food webs. Paleobiology 32, $1-19$.

30. Roopnarine, P. D., Angielczyk, K. D., Wang, S. C. and Hertog, R. 2007. Trophic network models explain instability of Early Triassic terrestrial communities. Proc. Roy. Soc. London B (in press).

31. Rosenzweig, M.L. 1975 On continental steady states of species diversity. In: Cody, M.L. and Diamond, J.M. (eds) Ecology and Evolution of Communities, pp. 121-140. Belknap Press, Cambridge, Massachusetts.

32. Sahney, S. and Benton, M. J. 2008. Recovery from the most profound mass extinction of all times. Proc. Roy. Soc. Lond. B 275, 759-765. 
33. Sepkoski, J. J. 1978. A kinetic model of Phanerozoic taxonomic diversity I. Analysis of marine orders. Paleobiology 4: 223-251.

34. Sepkoski, J. J. 1979. A kinetic model of Phanerozoic taxonomic diversity II. Early Phanerozoic families and multiple equilibria. Paleobiology 5: 222251.

35. Sepkoski, J. J. 1984. A kinetic model of Phanerozoic taxonomic diversity III. Post-Paleozoic families and mass extinction. Paleobiology 10: 246-267.

36. Smith, A.B. and Jeffery, C.H. 1998. Selectivity of extinction among sea urchins at the end of the Cretaceous period. Nature 392: 69-71.

37. Solé, R.V., Montoya, J.M. and Erwin, D.H. 2002a. Recovery from mass extinction: evolutionary assembly in large-scale biosphere dynamics. Phil. Trans. Roy. Soc. Lond. B 357: 697-707.

38. Solé, R.V., Alonso, D. and McKane, A. 2002b. Selforganized instability in complex ecosystems. Phil. Trans. Roy. Soc. Lond. B 357: 667-681.

39. Solé, R.V. and Bascompte, J. 2006. Selforganization in complex ecosystems. Princeton U. Press, Princeton.
40. Szatmáry, E. and Maynard Smith, J. 1997. From replicators to reproducers: the first major transitions leading to life. J. Theor. Biol. 187: 555-571.

41. Valentine, J.W. 1980. Determinants of diversity in higher taxonomic categories. Paleobiology 6: 44450 .

42. Valentine, J. W., and T. D. Walker. 1986. Diversity trends within a model taxonomic hierarchy and evolution. Physica 22D:31-42.

43. Valentine, J. W., and T. D. Walker. 1987. Extinction in a model taxonomic hierarchy. Paleobiology 13:193-107.

44. Wagner, P. J., Kosnik, M. A. and Lidgard, S. 2006. Abundance distributions imply elevated complexity of Post-Paleozoic Marine Ecosystems. Science 314, 1289-1292.

45. Williams, R. and Martinez, N. 2000. Simple rules yield complex food webs. Nature 404:180-3.

46. Wooton, J.T. 2005. Field parameterization and experimental test of the neutral theory of biodiversity. Nature 433: 309-312. 